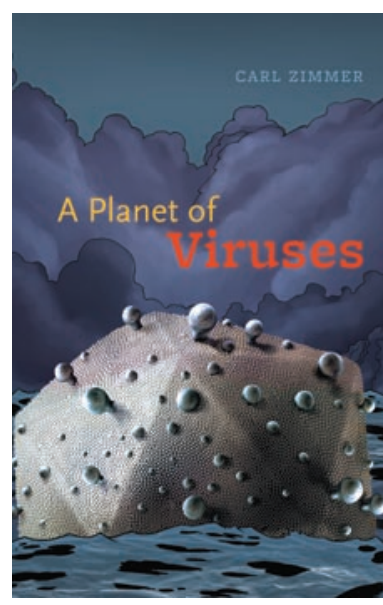

\section{A planet of viruses}

\author{
Carl Zimmer \\ University of Chicago Press. Chicago, Illinois, USA. 2011. \\ 128 pp. \$20.00. ISBN: 978-0-226-98335-6 (hardcover).
}

Reviewed by Lawrence T. Feldman

Department of Microbiology, Immunology and Molecular Genetics, David Geffen School of Medicine at UCLA, UCLA, Los Angeles, California, USA.

E-mail: LFeldman@mednet.ucla.edu
$\mathrm{V}$ iruses are all around us, in the water we drink, in the oceans we swim in, and in many unlikely places. Carl Zimmer starts his book of essays, A Planet of Viruses, by taking us deep into the Cave of Crystals in a remote province of Mexico. In a place in which there are enormous crystals but little apparent life, he shows that samples of the water taken in 2009 revealed the presence of millions of viruses. The story is typical of this charming little book, which is full of interesting anecdotes and contains many facts uncovered in just the last few years. This book is an easy read for the lay person, broken into small chapters, each featuring its own virus. Yet it was also fun and informative for me, for, while I knew much of this before, Zimmer was able to surprise me with a few fascinating nuggets I had not previously known.

In the introduction, we emerge from the Cave of Crystals to travel back in time to the classic discovery of tobacco mosaic virus by Beijerinck at end of the 19th century. Zimmer seamlessly introduces the idea of filtering of the virus to distinguish it from a bacterial cell, a concept you would find in any college course in virology.

Following this introduction to what a virus is, Zimmer divides his presentation into three sections. In Old Companions, we get a description of rhinovirus, the cause of the common cold, from a 3,500-year-old Egyptian medical text. This is followed by an interesting chapter on influenza virus, in which we get a very understandable description of the process of reassortment, somewhat unique to influenza, which contributes to the great genetic diversity of flu strains. Zimmer describes reassortment as a viral version of sex. In this theme, the third description is of human papillomavirus. Again we are treated to a story, this one about horns on rabbits, as Zimmer unwinds the story of Shope papilloma virus, discovered by Richard Shope of The Rockefeller University. We then learn about the human strains and zur Hausen's discovery that some of these cause cervical cancer in women.

In Everywhere, In All Things, Zimmer takes us into the world of bacteriophages, the viruses that infect bacterial cells. $\mathrm{He}$ recounts the story of Felix d'Herelle and his attempt during World War I to cure dysentery with phages. He then relates the historical scientific dispute between d'Herelle and Bordet, regarding whether the effects of phage were biological or chemical in nature, before describing the current attempts to use phages to kill bacteria. This section also covers the group of marine phages. One sentence contains the staggering number of phages in the ocean, a number with too many zeros to comprehend. I found myself trying to figure out what the number would be called. What comes after a billion $\left(10^{9}\right)$ is a trillion $\left(10^{12}\right)$, and after that is a quadrillion $\left(10^{15}\right)$. I won't spoil it by telling you how many logs short my guess was, but it was many. We also learn about the effects of phages on such processes as photosynthesis as well as cholera epidemics. The final group discussed are the endogenous retro- viruses, and we learn about all the viral junk DNA within our genome.

The final section is called The Viral Future and contains the discovery of HIV in Los Angeles in 1983 and the finding of West Nile virus in dead animals in the Bronx Zoo. Zimmer's writing is again instructive, as West Nile Virus is a classic example of a virus transmitted by insects (mosquitoes). This is followed by a description of the SARS epidemic that began in late 2002 and comments about Ebola virus in Africa and Nipah virus in Southeast Asia. Of course, no book on viruses would be complete without a description of smallpox virus, and Zimmer goes over the familiar stories of Jenner and the milkmaid, ending with the eradication of smallpox by a large vaccination program.

The epilogue contains a description of the discovery of Mimivirus, a virus of algae that is one hundred times of size of other viruses and may challenge the view that viruses are not living things.

All within one hundred pages or so, Zimmer treats us to a comprehensive series of anecdotes that reveal the diversity of virology on earth. From the study of oceanic samples to the finding of viral DNA in our lungs, from the viruses integrated into our chromosomes to those that cause cancer, the tales in this book will be of interest to anyone with an interest in science and are related in an easy-to-read style that requires no prior knowledge in biology. I would expect it to also be of interest to medical students and a wide range of health care professionals. It was a fun read. 